\title{
Recirculating linac free-electron laser driver
}

\author{
Peter H. Williams, ${ }^{*}$ Deepa Angal-Kalinin, David J. Dunning, James K. Jones, and Neil R. Thompson \\ STFC Daresbury Laboratory \& Cockcroft Institute, Warrington, Cheshire, WA4 4AD, United Kingdom
}

(Received 15 December 2010; published 17 May 2011; publisher error corrected 25 May 2011)

\begin{abstract}
This paper describes the design of a recirculating linac as a driver for the suite of seeded free-electron lasers (FELs) proposed in the UK New Light Source (NLS) project. The choice of superconducting technology for NLS is required in order to deliver bunches at high repetition rates up to $1 \mathrm{MHz}$. This raises the question of whether a shorter linac in recirculating mode can deliver the beam quality required for seeded FELs. To design such a facility, careful layout choices and optimizations must be made to ensure emittance growth is minimized. Effects leading to emittance dilution include chromatic transport terms, incoherent and coherent synchrotron radiation. The design outlined here is based on a modular philosophy to separate beam injection and extraction from a three stage compression scheme. The design uses many novel design concepts and optimizations to deliver the necessary high peak currents while preserving beam quality for seeded FELs. Start-to-end simulations including the FELs show that the necessary pulse coherence and output power can be provided from the beam thus generated.
\end{abstract}

DOI: 10.1103/PhysRevSTAB.14.050704

PACS numbers: 29.27.Eg, 29.27.Ac, 29.27.Bd, 29.27.Fh

\section{INTRODUCTION}

The New Light Source project (NLS) [1] was launched in April 2008 by the UK Science and Technology Facilities Council (STFC) to consider the scientific case and develop a conceptual design for a possible next generation light source. The facility will be based on a combination of advanced conventional laser and high harmonic generation (HHG) seeded free-electron laser (FEL) sources.

The NLS science case requires pulse repetition rates of up to $1 \mathrm{MHz}$, the accelerator is therefore based on a 1.3 GHz superconducting linac. Superconducting infrastructure necessitates comparatively large capital outlay and running costs. A recirculating linac design mitigates this in that a lower energy linac is required when the beam is accelerated through it multiple times. It is therefore important to assess whether it is possible to design a recirculating linac capable of delivering the challenging bunch parameters viz. a $200 \mathrm{pC}$ bunch with small emittances and energy spread with a peak current of more than $1 \mathrm{kA}$ to drive the FELs. The seeded nature also demands that bunch slice parameters remain constant over a time period of the order $100 \mathrm{fs}$ to take account of electron beam timing jitter with respect to the seed laser pulse.

The accelerator design described here takes the form of a two pass recirculating machine based on nine-cell TESLA-type accelerating cryomodules. The design of linac injection, extraction, and recirculating transport sections are described, together with post-linac components.

\footnotetext{
*peter.williams@stfc.ac.uk
}

Published by the American Physical Society under the terms of the Creative Commons Attribution 3.0 License. Further distribution of this work must maintain attribution to the author(s) and the published article's title, journal citation, and DOI.
The design uses automation and optimization techniques both in transverse optics design and the longitudinal bunch compression scheme. Finally, full start-to-end tracking simulations are performed from the cathode to the resultant FEL radiation pulse and demonstrate the capability of a recirculating linac to fulfill the stringent requirements needed to drive a suite of seeded FELs.

Additional advantages of a recirculating linac include that any tunneling or shielded building above ground would be shorter in comparison to a single pass machine (however, in parts it would need to be wider); and that one is able to extract beams of different energies from one location, enabling FELs resonant at different wavelengths to operate efficiently. A final advantage is that a natural upgrade path is established without the need to add accelerating modules; instead additional recirculation paths can be constructed.

Design issues that are additional to those of a single pass machine include the following: It is necessary to combine and separate beams of different energies, this leads to more complex transverse optics. The bunch compression and linearization scheme is restricted in sections with multiple beam energies. Arcs will be required to bend the beam by $360^{\circ}$; incoherent synchrotron radiation within these will lead to emittance degradation. Coherent synchrotron radiation (CSR) will be emitted on any transverse manipulation when the bunch is short, which will also lead to emittance degradation.

\section{FACILITY DESIGN}

The NLS accelerator design specifications are to produce electron bunches of charge $200 \mathrm{pC}$ with less than $1 \mathrm{~mm}$ mrad transverse slice emittance, peak current above $1 \mathrm{kA}$, and slice energy spread less than $5 \times 10^{-4}$ at an 
energy of $2.2 \mathrm{GeV}$. This is achieved through a $200 \mathrm{MeV}$ injector followed by two recirculation passes of a $1 \mathrm{GeV}$ main linac [2]. An alternative philosophy would be to view recirculation as a way to extend the energy of a single pass machine of $2.2 \mathrm{GeV}$; this scenario is not addressed in this paper although it could be revisited in the context of a future upgrade. Other configurations that were considered and rejected include placing the linac in both legs; and a dog-bone topology with a $360^{\circ}$ arc. These introduced additional issues without significant benefit.

In order to maintain maximal benefit from recirculation, one should inject into the main linac at as low an energy as possible. However, this aim must be traded off against considerations arising from the method of longitudinally compressing the bunch, i.e., the interplay between off crest rf acceleration, which imparts an energy-time correlation on the bunch, and magnetic chicanes, where transverse dispersion is used to allow the higher energy back of the bunch catch up with the front. As off crest rf acceleration is used, the energy-time correlation is not linear, and the longer the bunch is on entering the main linac, the greater the amount of rf curvature seen by the bunch. This curvature impairs the ability to compress the bunch, thereby limiting peak current and subsequent lasing in the FELs. The standard method to mitigate this is to utilize a higherharmonic rf cavity to linearize the longitudinal phase space. The maximum voltage achievable in this higherharmonic system then becomes the limiting factor. The bunch needs enough compression on entry to the main linac to compensate for the curvature within the design parameters of the harmonic system. An additional consideration is the deceleration imparted by the higher-harmonic system must be performed from a sufficiently high initial energy that space charge effects cannot significantly affect the beam during subsequent injection transport to the main linac. It is possible to perform this compression prior to main linac injection due to the far weaker longitudinal wakefields in superconducting accelerating sections as compared with warm accelerating sections.

One-dimensional longitudinal phase space tracking was utilized to optimize on the injection energy given the above constraints. This process leads to the proposed injector design illustrated in Fig. 1; two accelerating cryomodules are positioned in the injector, followed by a third harmonic system. The injection energy is thus approximately
$200 \mathrm{MeV}$. In order to reach the design energy therefore requires a $1 \mathrm{GeV}$ main linac. The ratio of beam energies at injection is 6 and that at extraction is 1.8; this is sufficiently far from unity to enable an efficient extraction system design.

In the following sections the individual sections of the accelerator are described in detail. The machine lattice and tracking simulations have been performed using ELEGANT [3]. For extensive optimizations this was interfaced with MATHEMATICA $^{\mathrm{TM}}$. Optics design and matching was performed using MAD and TRANSPORT [4]. In referring to the bunch compression scheme, the first order momentum compaction of a section (also referred to as longitudinal dispersion or cumulative transport matrix element $R_{56}$ ) is negative if a negatively chirped bunch is compressed. In other words, higher energy particles are at the back of the bunch and are allowed to catch up with the front. Thus, a standard four dipole compression chicane has a negative $R_{56}$.

Throughout, the design philosophy is to minimize any bunch compression from components not dedicated for that purpose, in other words separating as much as possible transverse and longitudinal manipulations of the phase space of the bunch. In this way, one retains the ability to tailor the final bunch profile in a flexible way.

\section{A. Components and optics}

A large correlated energy spread (chirp) is required for magnetic bunch compression to be effective. Such a beam is sensitive to higher order transport matrix elements that involve energy dependence, for example chromaticity and second order dispersion. These will cause emittance increase due to the imprinting of curvatures in the phase space of the relevant terms. It is therefore important that all sections of the recirculating linac are well behaved at both first and second order in the transfer matrices. To achieve this, a novel brute force method of chromatic and geometric aberration correction has been developed. A Nelder-Mead simplex optimization algorithm [5] is used to minimize the horizontal and vertical emittances and $T_{566}$ using the number, strengths, and positions of sextupoles as the optimization variables in relevant sections. This must be performed for the injection achromat, linac-to-arc, and arc-to-linac matching sections.

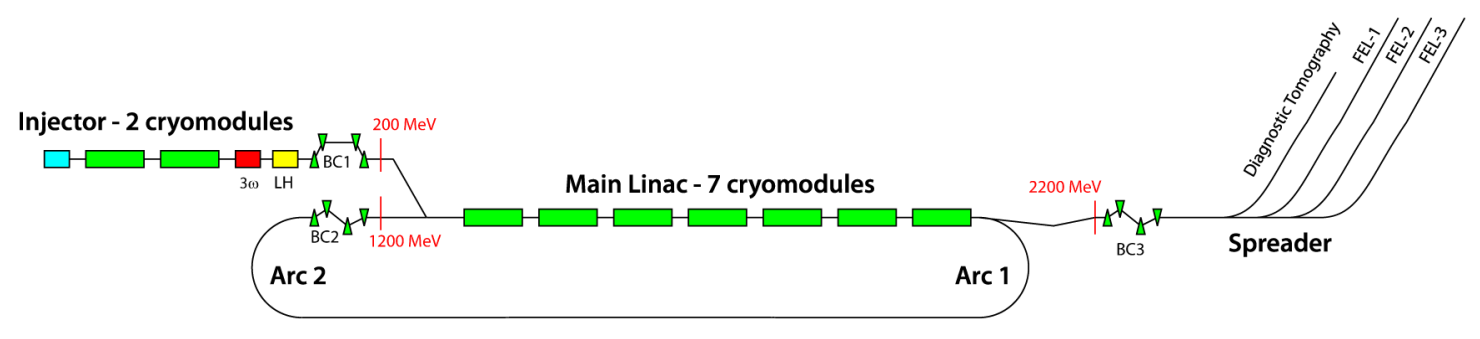

FIG. 1. Schematic layout of the NLS recirculating linac. 


\section{Injection straight and first bunch compressor}

The photoinjector is followed by two cryomodules, the first of whose cavities are fed from individual power supplies to enable independent phase and voltage control. This allows an effective emittance compensation scheme to be applied while the bunch is in the nonrelativistic regime. The configuration is simulated using ASTRA [6] which indicates that normalized transverse emittances of $0.3 \mathrm{~mm}$ mrad are achieved with a bunch length of 15 ps (FWHM) [7]. The second cryomodule takes the energy from 135 to $265 \mathrm{MeV}$. A third harmonic cryomodule, consisting of eight cavities, then overlinearizes the bunch to compensate for rf curvature imposed by both upstream and downstream off crest acceleration. This reduces the centroid energy to $220 \mathrm{MeV}$.

The two injector accelerating modules and linearizing module are situated in conjunction in order to provide for efficient cryogenic interconnection design for $\mathrm{cw}$ operation. Subsequent to these is a warm section with a small chicane and laser system arranged to form a "laser heater." This is intended to provide a method of increasing the uncorrelated slice energy spread within the bunch [8]. The beam now enters the first magnetic compression chicane. This is a four dipole symmetric C-type design, whose compression properties are described in Sec. II B.

\section{Injection achromat}

To inject into the main linac, a number of beam dynamics and layout restrictions need to be satisfied. The transport must be achromatic and isochronous in order not to restrict the overall bunch compression scheme. Necessarily, the system shares the final dipole with the high energy returned beam in an energy ratio of 6:1, vertical focusing arising from the nonoptimal edge angle of this dipole is therefore another restriction that must be dealt with carefully. In order to minimize the impact of this section on the building width, the injection system is parallel to the linac, therefore the total angle of the system is zero. With an energy spread of $\mathcal{O}(3 \%)$, the injection achromat requires the strongest correction to the second order transfer matrices of the entire machine. Finally, the optics functions entering the linac must be such that control of them along the linac is possible using the same focusing elements that simultaneously affect the high energy beam.

In order to cope simultaneously with these restrictions, a carefully considered first order optics design has been employed with additional correction at second order. The high energy chicane needs to have a minimum dipole angle of $-2^{\circ}$ (at $1.2 \mathrm{GeV}$ ) to allow merging of the injected beam. This angle becomes $12^{\circ}$ for the injected beam (at $200 \mathrm{MeV}$ ). A symmetric achromatic system with two dipoles of opposite polarities is not suitable here due to the asymmetric edge angle of the second dipole, large optics functions, and dispersion functions as well as the nonisochronicity of such a system. At large values of the optics functions, the lattice becomes unacceptably sensitive to
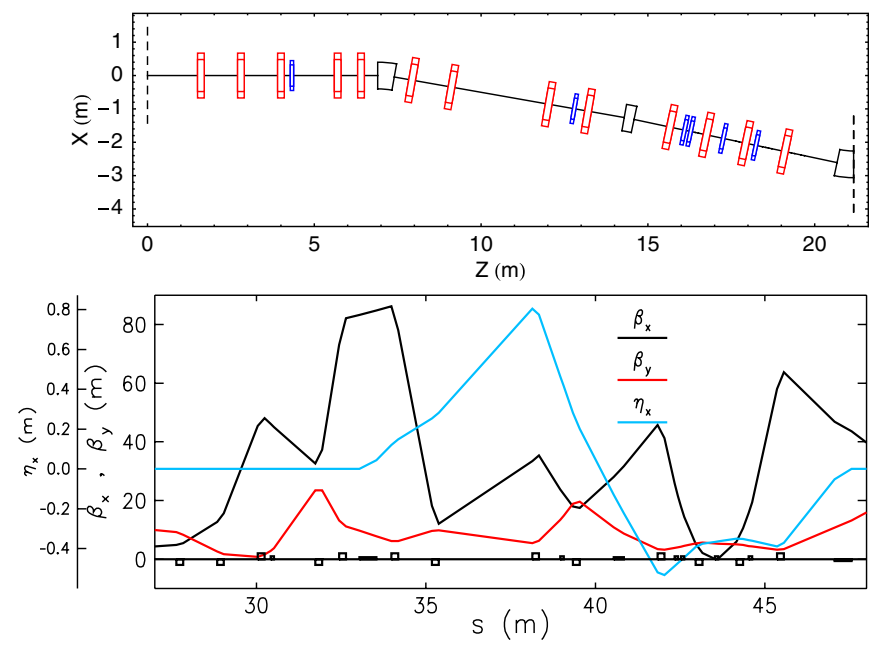

FIG. 2. Top: Floor layout of the injection achromat, sextupoles shown in blue. Bottom: Optics and dispersion functions in injection achromat.

second order terms. In order to minimize these effects, the chosen design uses two dipoles in addition to the merge dipole (of angles $+11^{\circ}$ and $+1^{\circ}$, respectively) and eight quadrupoles in the dispersive section to make the overall system achromatic and isochronous with reasonable optical and dispersion functions. Five quadrupoles preceding the dispersive section are used to match the Twiss parameters to the linac. The floor layout of the injection achromat and corresponding optics is shown in Fig. 2. On tracking a bunch through this system, it is seen that chromatic aberrations are introduced and the emittance increases due to these nonlinearities. To analyze this, the second order matrix elements involving energy, i.e., the $T_{i j 6}$ terms are analyzed. Longitudinally, $T_{566}$ is the relevant parameter, but transversely looking at all the terms is not illustrative as they are not independent. It is preferable to recast in terms of the variation of the Twiss parameters and dispersion with respect to energy. Thus, for the horizontal plane, the chromatic amplitude functions are defined as

$$
\begin{gathered}
W_{x} \equiv \sqrt{a_{x}^{2}+b_{x}^{2}} \quad \text { where } b_{x}=\frac{1}{\beta_{x}} \frac{d \beta_{x}}{d \delta}, \\
a_{x}=\frac{d \alpha_{x}}{d \delta}-\frac{\alpha_{x}}{\beta_{x}} \frac{d \beta_{x}}{d \delta}
\end{gathered}
$$

and the chromatic derivative of dispersion

$$
\frac{d D_{x}}{d \delta} \equiv \frac{d^{2} x}{d \delta^{2}}
$$

with analogous definitions for the vertical plane. Figure 3 shows the chromatic amplitude functions. It can be seen that these become large by the end of the injection achromat, particularly in the horizontal plane. Figure 3 also shows the chromatic derivative of dispersion; this is seen to diverge after the achromat. The second order optimization procedure has settled on six sextupoles with five in the 

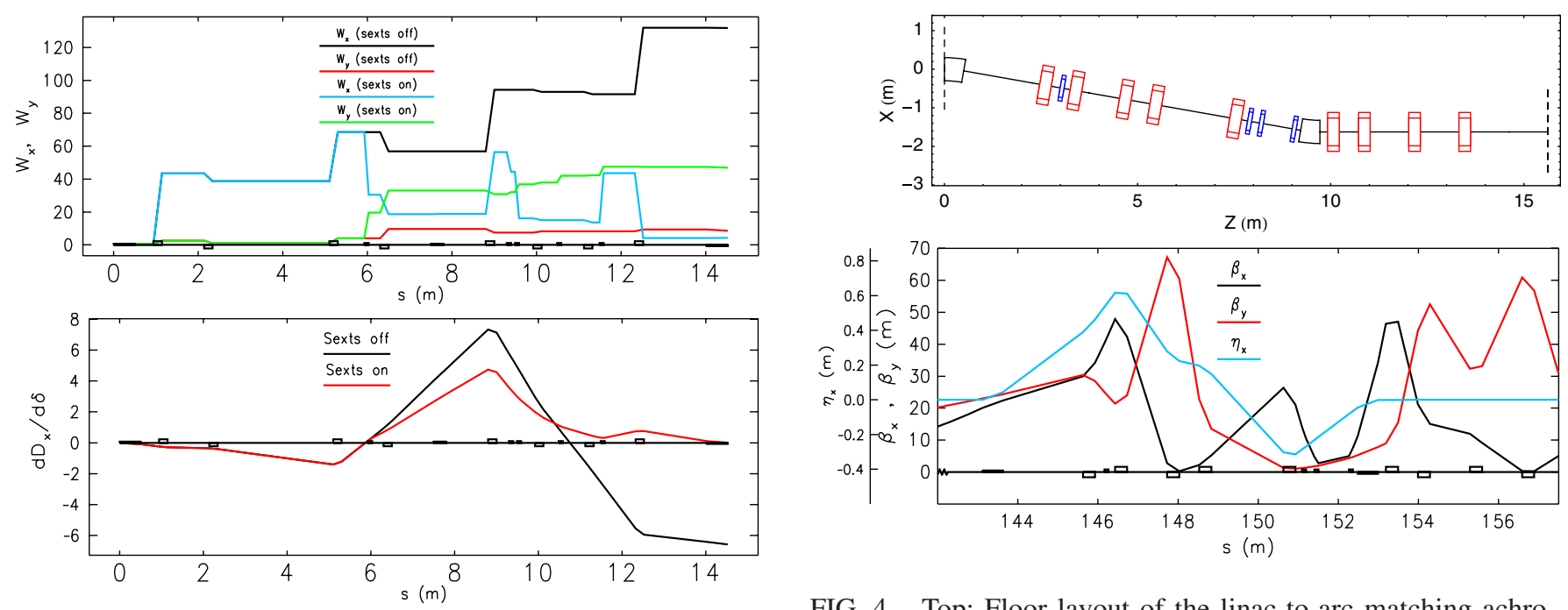

FIG. 3. Top: Chromatic amplitude functions in the injection achromat before (black, red) and after (blue, green) correction. Bottom: Chromatic derivative of dispersion before (black) and after (red) correction.

dispersive region and one in the preceding matching section. The sextupoles in the dispersive region control the chromatic second order functions; however, in doing this some geometric second order terms become large; this is corrected by placing one sextupole in the dispersion free section. The chromatic amplitude functions are reduced by a factor of 10 in the horizontal plane although at the cost of an increase in the vertical plane (this is unimportant as there is zero vertical dispersion). The chromatic derivative of dispersion is fully corrected. The value of $T_{566}$ after correction is -0.35 , a fourfold reduction from the uncorrected value.

\section{Achromat matching linac to arc at $1.2 \mathrm{GeV}$}

The dipole located at the exit of the main linac separates the 1.2 and $2.2 \mathrm{GeV}$ beams. Two lines that transport each beam without conflicting with each other are designed. The 1.2 GeV line must then match into the first arc. The separation dipole must separate the two beams transversely in a sufficiently short distance to allow insertion of independent optics elements in the two transport lines. However, the angle should not be too large as this would imply large optics functions and dispersion, which in turn implies sensitivity to higher order terms. A $10^{\circ}$ angle for the $1.2 \mathrm{GeV}$ beam was chosen to achieve this. Figure 4 shows floor layout and optics for this system. The optical functions are matched to the arc Twiss parameters and are kept below $80 \mathrm{~m}$ with maximum dispersion of $0.6 \mathrm{~m}$. The system is achromatic and though not isochronous it has been optimized to keep $R_{56}$ small ( $+5 \mathrm{~mm}$ ). This is sufficiently small in comparison to the bunch compressors that the longitudinal tuning of the machine remains almost independent of the transverse transport.

FIG. 4. Top: Floor layout of the linac-to-arc matching achromat, sextupoles shown in blue. Bottom: Optics and dispersion functions in linac-to-arc matching achromat.
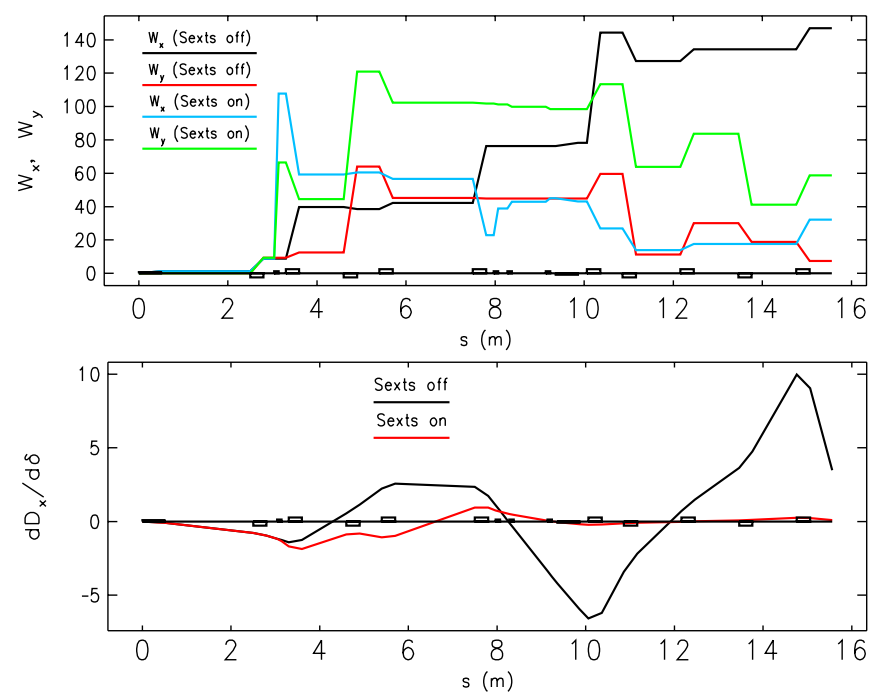

FIG. 5. Top: Chromatic amplitude functions in the linac-to-arc matching achromat before (black, red) and after (blue, green) correction. Bottom: Chromatic derivative of dispersion before (black) and after (red) correction.

As with the injection system, the optimization method was applied to correct the chromatically induced emittance growth resulting in Fig. 5. The horizontal chromatic amplitude function is reduced by a factor of 5; this is made at the expense of a commensurate increase in the vertical plane. The chromatic derivative of dispersion is reduced by an order of magnitude.

\section{Arcs and return path}

The requirements for the arcs are to turn the beam by $180^{\circ}$ in each arc with as small a footprint as possible, for economic reasons. However, the beam should be degraded as little as possible. A number of preexisting designs were 
evaluated as to their suitability for NLS. Of these the arc design for the BESSY-FEL fulfilled all requirements [9]. This consists of four triple-bend achromat structures, each bending the beam by $45^{\circ}$. Two sextupole families are used to control $T_{566}$ to ensure that the requirements on the third harmonic linearization module in the injector are not too onerous.

The optics at the exit of the arc is matched into a long transport line that brings the beam to the start of the second arc. This line utilizes a simple focus-drift-defocus-drift (FODO) channel with $45^{\circ}$ phase advance. This was chosen with the intention of inserting a tomography diagnostics section to reconstruct the six-dimensional phase space of the bunch after acceleration and transport in the arc. Since this FODO does not affect the beam dynamics, the phase advance can easily be reduced to decrease the number of components for cost benefit. Within this $140 \mathrm{~m}$ section it is intended to insert an additional path length correction system [10]. This will enable the total path length of the return transport to be varied over one complete rf wavelength $(23 \mathrm{~cm})$. The system will comprise a pair of second order achromatic doglegs connected with a short bellows section. The path length variation will be achieved independently of all transverse beam properties, but will introduce a small $(1-2 \mathrm{~mm}) R_{56}$ dependence as the path length is changed; this can be easily compensated for in the second bunch compressor. This has not yet been implemented in the simulated machine, though independent control of the linac phase in the second pass has been assumed.

The beam is then matched into the second $180^{\circ}$ arc and emerges transversely displaced by $2 \mathrm{~m}$ with respect to the main linac. It has been chosen to insert an exact reverse of the linac-to-arc matching achromat in order to bring the beam collinear to the linac. The reason for this is to ensure
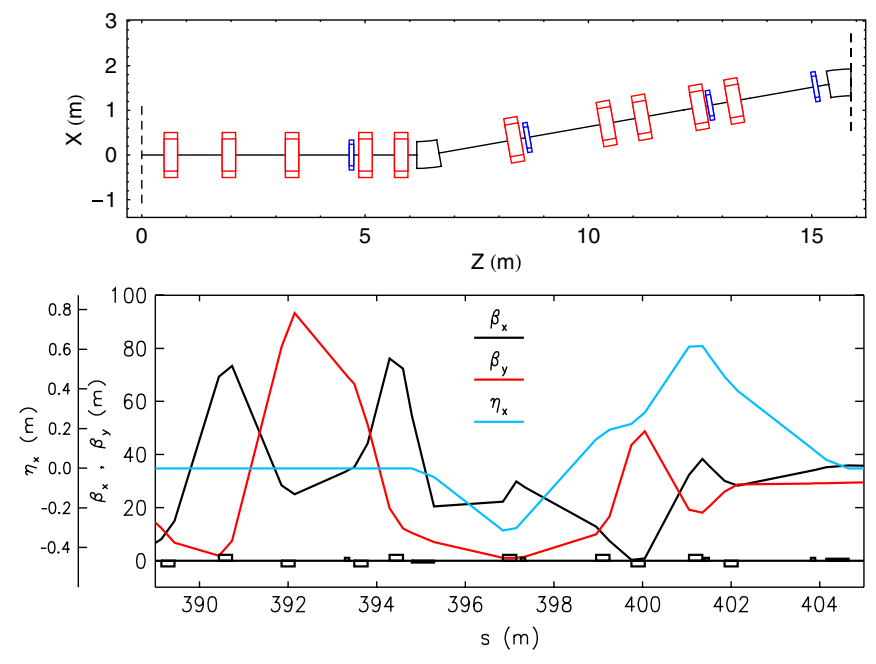

FIG. 6. Top: Floor layout of the arc-to-linac matching achromat, sextupoles shown in blue. Bottom: Optics and dispersion functions in arc-to-linac matching achromat.
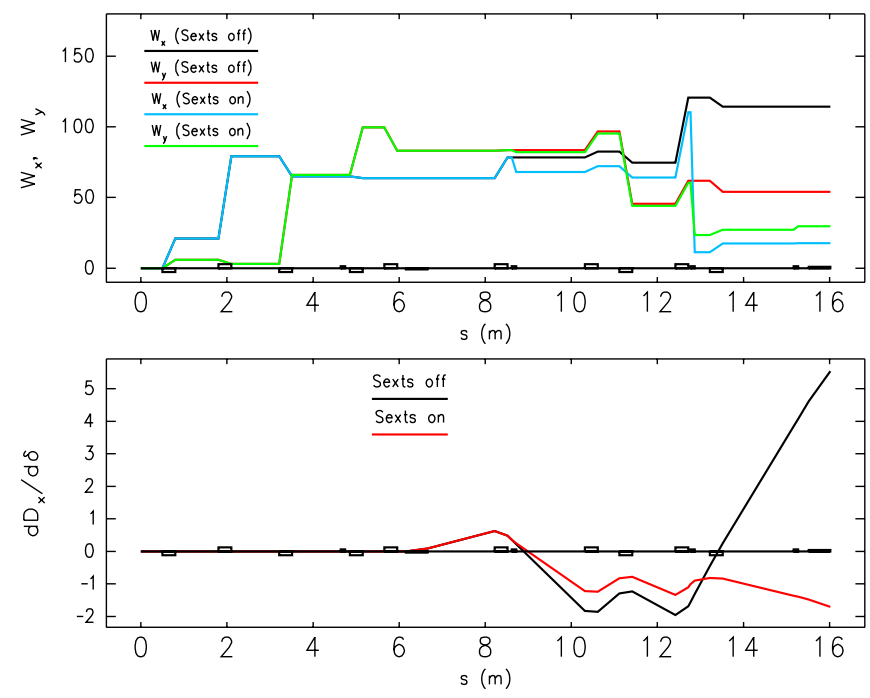

FIG. 7. Top: Chromatic amplitude functions in the arc-to-linac matching achromat before (black, red) and after (blue, green) correction. Bottom: Chromatic derivative of dispersion before (black) and after (red) correction.

that identical magnets exist in the two arcs and extraction enabling the number of independent power supplies to be reduced. A separate sextupole optimization is required from the one performed on the linac-to-arc system. The reason for this is the previously mentioned choice to correct for $T_{566}$ in the arcs at the expense of the chromatic terms. This gives the layout shown in Fig. 6 which has four sextupoles, three in the dispersive region and one in the preceding matching section to correct for geometric second order terms induced through the chromatic correction. The effect of these corrections is shown in Fig. 7.

\section{Second bunch compressor and reinjection}

The second bunch compressor is located before reinjection into the main linac. As this is after the arcs, it is possible to compress the bunch strongly. However, for bunches shorter than 1 ps full width (FW), the CSR emittance growth can be significant. Both C-type and S-type chicanes were assessed for their suitability at this location, with the choice of S-type being due to the need to minimize CSR emittance growth. As with the first compressor the horizontal beta function is minimized in the final dipole to further suppress CSR emittance growth. The beam then passes through the high energy path of the injection merger. This is a weak C-type four dipole chicane with optimal edge angles for this $1.2 \mathrm{GeV}$ beam (the nonoptimal angle was chosen to be seen by the injected beam).

\section{Linac second pass}

The linac consists of seven cryomodules (each of eight nine-cell cavities) separated by quadrupole doublet matching sections. An optics cell thus has its boundary at the midpoint between the two quadrupoles. In order to 

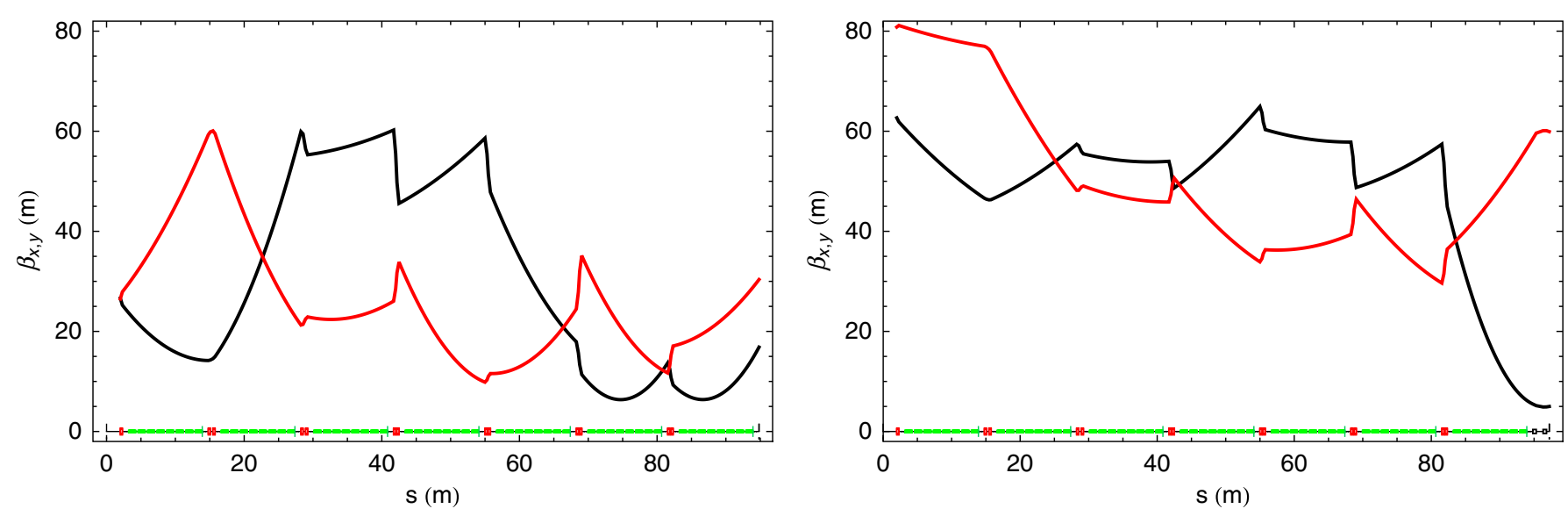

FIG. 8. Simultaneous optics functions for first (left) and second (right) pass of main linac.

minimize CSR induced emittance growth in the extraction dipole at the end of the main linac, the horizontal beta function at this dipole must be minimized. Failure to do this was shown to increase the emittance by an order of magnitude. This is not trivial as the quadrupoles in the linac focus the first pass lower energy beam more strongly. To address this problem a dual optimization of Twiss parameters was performed by the interfacing of MAD with MATHEMATICA. A Nelder-Mead simplex optimization algorithm was employed with variables being the linac quadrupole currents. These were scaled to strength $(k)$ values for both passes of the linac and Twiss parameters found for both cases before constructing the optimization penalty function. Constraints imposed were maximum and minimum acceptable beta function values over the entire linac length, maximum acceptable alpha function values at the exit of the extraction dipole, and a strong minimization requirement on the horizontal beta function value at the exit of the extraction dipole in the $2.2 \mathrm{GeV}$ case. Figure 8 shows the optimized optics functions for the two energy beams simultaneously passing through the linac. In performing the above optimization, care must be taken when implementing the results into the ELEGANT tracking simulation. ELEGANT and MAD use different approximations in the calculation of $\mathrm{rf}$ focusing. This effect arises due to off crest acceleration and therefore the beam sees not only $\boldsymbol{E}_{z}$ but also $\boldsymbol{E}_{r}$ and $\boldsymbol{B}_{\phi}$; this is required in order to fulfill the Maxwell equations $\nabla \cdot E=0$ and $\nabla \times B=\partial E / \partial t$. MAD implements an approximate analytic solution of the resulting transverse equation of motion known as the Chambers model [11], but only does so on entry and exit of a cavity. ELEGANT uses the more sophisticated Rosenzweig-Serafini model [12] which directly gives the transfer matrix of an arbitrary standing wave mode including harmonic content; this reduces to the Chambers model for pure $\pi$-mode cavities. However, ELEGANT calculates this effect not just at entry and exit, but also through the entire cavity body. This leads to a small difference in the Twiss parameters at the end of the main linac between the two programs.
A slight rematching of $1.2 \mathrm{GeV}$ parameters was done to cope with this difference.

\section{Extraction at $2.2 \mathrm{GeV}$ and third bunch compressor}

The requirements to be fulfilled for the $2.2 \mathrm{GeV}$ extraction system are that the optics functions and dispersion should not become large and bends should be shallow. This will minimize the expected CSR induced emittance growth. The line should be achromatic and as close to isochronous as practicable. Chromatic considerations are not as important as the typical energy spread at this point is only $0.6 \%$. Figure 9 shows the floor layout of the extraction region, this includes the linac-to-arc matching achromat in order to demonstrate that there is no clash. The third bunch compressor follows the extraction section, again an S-type design has been chosen in order to minimize CSR induced emittance growth. A preceding matching section ensures
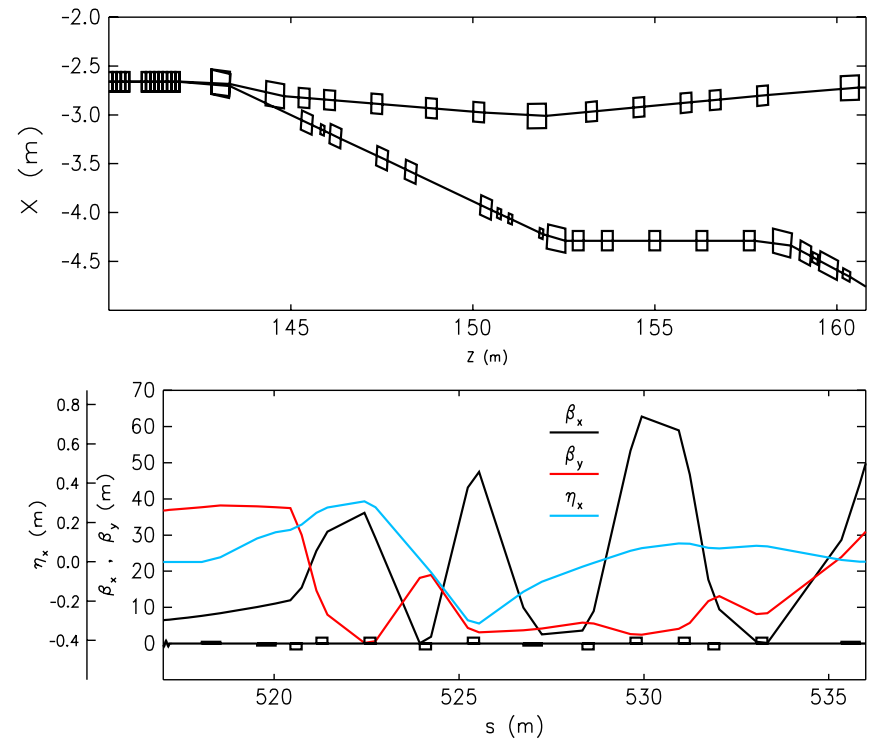

FIG. 9. Top: Floor layout of the extraction region. Bottom: Optics and dispersion functions through the extraction system. 


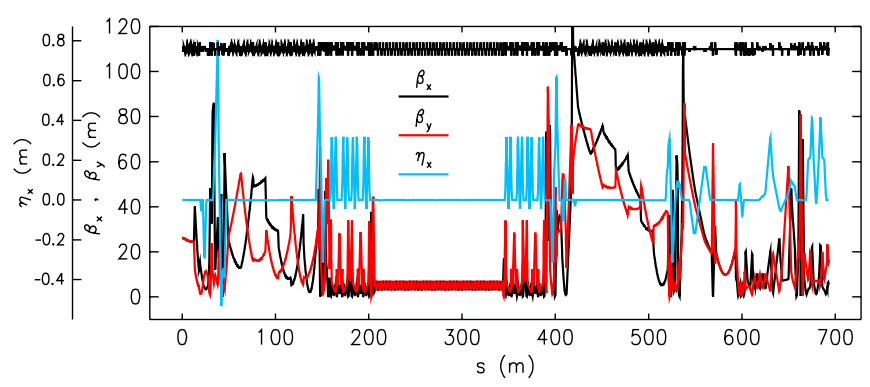

FIG. 10. Optics functions and dispersion for the recirculating linac including collimation and spreader.

that the horizontal beta function is minimized in the final dipole.

After the third compressor, the beam is matched to a post-linac section. This consists of collimation and beam spreader sections, the design of which is described elsewhere [13]. Figure 10 shows the optics for the entire machine from the second injection module to the end of the spreader.

\section{B. Longitudinal optimization}

The longitudinal bunch compression scheme is predominantly governed by the strengths of the three bunch compressors, the off crest chirp imparted in the injector and first and second passes of the main linac, and the gradient and phase of the third harmonic linearizing module. Tuning these to give the correct final bunch properties is thus an eight-dimensional optimization problem. Defining the constraints is far from straightforward. NLS is a high repetition rate capable suite of seeded FELs. Therefore the bunch must arrive at the FELs in coincidence with a HHG seed laser pulse. In order to allow for jitter between the bunch and the laser seed, the slice properties of the bunch should be as constant as possible over some time window [14]. An initial attempt was made to perform this optimization using the Nelder-Mead simplex method. This was found to be inadequate. The Nelder-Mead simplex method operates by constructing an $\mathrm{N}$-dimensional derivative in the optimization variables by stepping up and down in each variable, then calculating a penalty function at each point. The new solution is then the point in variable space with the smallest penalty function. Thus, it moves "downhill" until it reaches a minimum. However, with highly nonlinear problems such as that being dealt with here, it tends to become stuck at a local minimum. It was decided to abandon this method and utilize the Luus-Jaakola pseudorandom global minimum search algorithm [15]. In this procedure, a volume of variable space is defined and points selected pseudorandomly within it. The penalty function is calculated for each point and the volume of variable space is recentered on that point. The volume is then reduced by some factor and the procedure repeated. The advantage of Luus-Jaakola over Nelder-Mead is the pseudorandom element; this ensures that the optimization does not get stuck at a local minimum but rather explores the entire variable space to find the global minimum. Applying this to the recirculating linac, each point corresponds to a specific machine. To calculate the penalty function, the bunch is tracked through this machine and the final bunch properties are analyzed. Practically, the multiple node cluster dl1.nwgrid.ac.uk which is part of the UK NorthWest Grid Infrastructure has been used. This machine consists of 96 nodes, each having two $2.4 \mathrm{GHz}$ dual core CPUs (total 384 cores), each node having $16 \mathrm{~Gb}$ memory with communications between nodes provided by an InfiniPath high bandwidth interconnect. Each machine is submitted as a job, with each job being run in parallel on a number of computational cores. Typically, 50 machines were tracked simultaneously on four cores each. A typical 100000 macroparticle track took 4 minutes to run. After each machine population had tracked, the results were analyzed using MATHEMATICA. This calculates the penalty function, populates the new volume, and submits to the cluster.

The definition of the penalty function is the crucial component in order to obtain a successful optimization. A degree of trial and error was needed to specify a mutually consistent and suitably weighted set of constraints, these are: (1) mean current (binned in 5 fs slices) over a contiguous $100 \mathrm{fs}$ slice to be greater than $1 \mathrm{kA}$; (2) minimum current (binned in $5 \mathrm{fs}$ slices) in that same $100 \mathrm{fs}$ slice to be greater than $950 \mathrm{~A}$; (3) standard deviation of current (binned in $5 \mathrm{fs}$ slices) in that same $100 \mathrm{fs}$ slice to be less than $50 \mathrm{~A}$; (4) rms bunch length to be less than $400 \mathrm{fs}$; (5) of the central $80 \%$ of the bunch binned by energy, the slice energy spread of all 5 fs slices to be less than

TABLE I. Optimized machine parameters. Phases are with respect to crest.

\begin{tabular}{lcr}
\hline \hline Optimized section & Variable & \multicolumn{1}{c}{ Value } \\
\hline $3 \omega$ linearizer & $E$ & $16 \mathrm{MV} / \mathrm{m}$ \\
$3 \omega$ linearizer & $\phi$ & $+160.9^{\circ}$ \\
Injector & $\phi$ & $-29.1^{\circ}$ \\
Linac first pass & $\phi$ & $-11.8^{\circ}$ \\
Linac second pass & $\phi$ & $+2.3^{\circ}$ \\
Bunch compressor 1 & $R_{56}$ & $-96.7 \mathrm{~mm}$ \\
Bunch compressor 2 & $R_{56}$ & $-80.9 \mathrm{~mm}$ \\
Bunch compressor 3 & $R_{56}$ & $-23.9 \mathrm{~mm}$ \\
\hline Fixed section & & \\
\hline Laser heater chicane & $R_{56}$ & $-4.9 \mathrm{~mm}$ \\
Injection achromat & $R_{56}$ & $0 \mathrm{~mm}$ \\
Linac-to-arc matching & $R_{56}$ & $+5 \mathrm{~mm}$ \\
Arc (two identical instances) & $R_{56}$ & $-18.65 \mathrm{~mm}$ \\
Arc-to-linac matching & $R_{56}$ & $+5 \mathrm{~mm}$ \\
Reinjection chicane & $R_{56}$ & $-1.6 \mathrm{~mm}$ \\
Extraction & $R_{56}$ & $-0.002 \mathrm{~mm}$ \\
Collimation & $R_{56}$ & $+0.26 \mathrm{~mm}$ \\
Spreader & $R_{56}$ & $0 \mathrm{~mm}$ \\
\hline \hline
\end{tabular}


$1.2 \times 10^{-4}$; (6) the 5 fs current slices two away on either side of the central slice in time must be such that the energy chirp ensures the back of the bunch has higher energy

The combination of these ensures a high peak current that is optimized to be as flat as possible in time over $100 \mathrm{fs}$, while keeping the bunch chirped in the correct direction to avoid passing through the minimum bunch length and "folding over" in longitudinal phase space. Table I shows the final optimized values and also lists momentum compactions for all other fixed sections. On completion of this optimization, a production run was initiated to produce $5 \times 10^{6}$ macroparticles to inspect the properties of the bunch manually and provide input for the time-dependent FEL simulations.

\section{Evolution of longitudinal phase space}

It is instructive to give a detailed commentary on the evolution of the longitudinal phase space. Table II summarizes the energy, bunch length, and total energy spread at critical locations in the machine. From this it can be seen that most compression occurs at the second bunch compressor; this gives confidence that the optimization procedure is working correctly. It has chosen to keep the bunch relatively long when passing through the arcs and only then to approach full compression. Figure 11 (top) shows the longitudinal phase space after each transformation in the injector. The bunch emerges from the second accelerating cryomodule at $262 \mathrm{MeV}$ with a FW energy spread of $6 \%$ and FW bunch length of 18 ps (black). After the third harmonic module its energy is lowered to $228 \mathrm{MeV}$ with FW energy spread of $2.8 \%$ and the imprinted curvature has been reversed (red). After the first bunch compressor the FW bunch length has been reduced to $5 \mathrm{ps}$ (blue). After the injection achromat the FW bunch length has increased slightly to $5.5 \mathrm{ps}$ due to removal of some curvature by the $T_{566}$ of this section (green). Figure 11 (middle) shows the longitudinal phase space after each transformation in the first pass of the machine. The bunch emerges from the linac-to-arc matching achromat at $1227 \mathrm{MeV}$ with a FW energy spread of $1.5 \%$ and a FW bunch length of $6 \mathrm{ps}$ (black). After both arcs and the transport between them, the FW bunch length is 4.5 ps due to compression from the arcs (red). After the arc-to-linac matching achromat the FW bunch length is lengthened slightly to 5 ps (blue). After bunch compressor 2 the FW bunch length is $900 \mathrm{fs}$

TABLE II. Bunch length and relative energy spread at critical points.

\begin{tabular}{lrrc}
\hline \hline Section & \multicolumn{1}{c}{ Energy } & $\sigma_{z}(\mathrm{FW})$ & $\sigma_{\delta}(\mathrm{FW})$ \\
\hline Start of tracking & $136 \mathrm{MeV}$ & $18 \mathrm{ps}$ & 2.8 \\
After BC1 & $228 \mathrm{MeV}$ & $5 \mathrm{ps}$ & $3.5 \%$ \\
Matching to arc 1 & $1227 \mathrm{MeV}$ & $6 \mathrm{ps}$ & $1.5 \%$ \\
After BC2 & $1227 \mathrm{MeV}$ & $900 \mathrm{fs}$ & $1.5 \%$ \\
After BC3 & $2244 \mathrm{MeV}$ & $350 \mathrm{fs}$ & $0.6 \%$ \\
\hline \hline
\end{tabular}
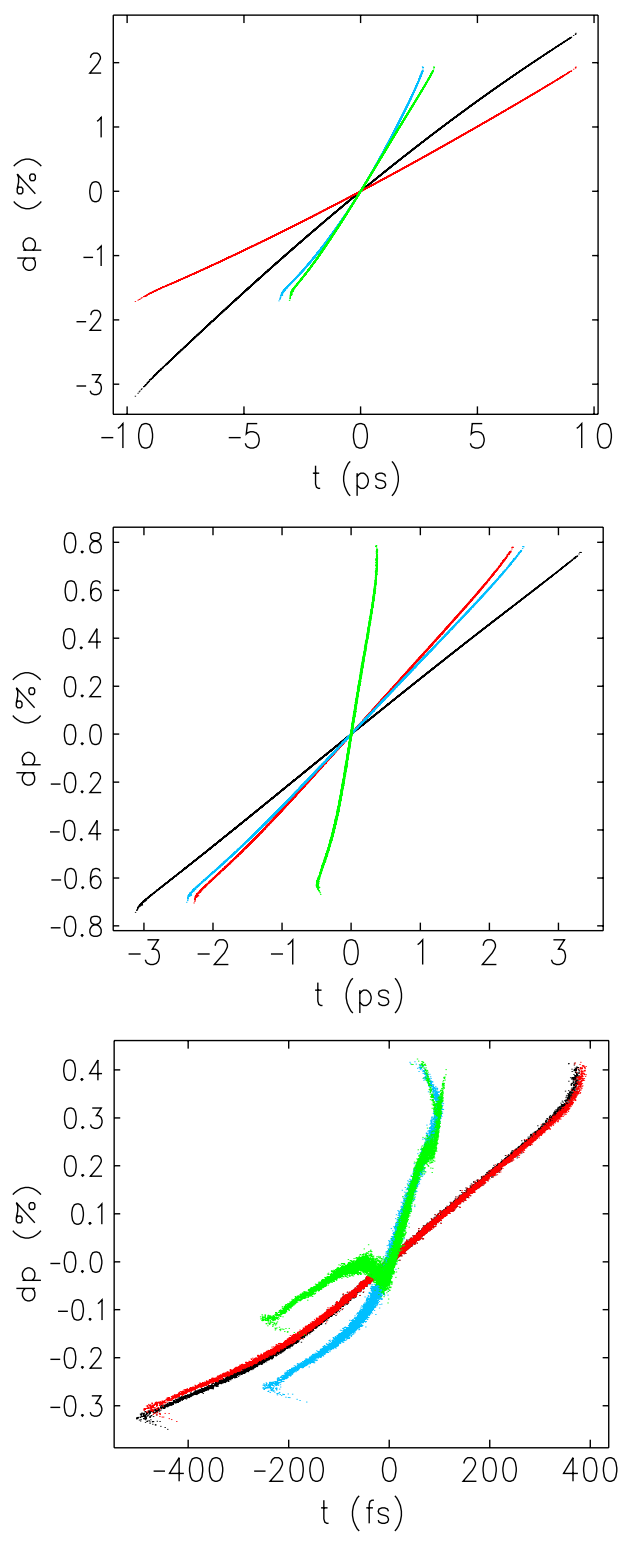

FIG. 11. Longitudinal phase space at progressing through the linac. Top: Sections at $228 \mathrm{MeV}$ (black-after second module; red-after third harmonic cavity; blue-after first bunch compressor; green - after injection achromat). Middle: Sections at $1227 \mathrm{MeV}$ (black-after linac-to-arc achromat; red-at exit of arc 2; blue - after arc-to-linac matching achromat; green-after bunch compressor 2). Bottom: Sections at $2244 \mathrm{MeV}$ (blackafter linac second pass; red-after extraction; blue-after bunch compressor 3; green-after spreader and on entrance to FEL).

(green). Figure 11 (bottom) shows the longitudinal phase space after each transformation on extraction at $2244 \mathrm{MeV}$. The initial FW energy spread is $0.7 \%$ and FW bunch length is $900 \mathrm{fs}$ (black). After the extraction section it is shortened to $850 \mathrm{fs}$ due to the slight compression (red). After the third bunch compressor the FW bunch length is reduced to $350 \mathrm{fs}$; additionally the FW energy spread is reduced to $0.6 \%$ by the CSR decreasing the energy of the back of the bunch (blue). 


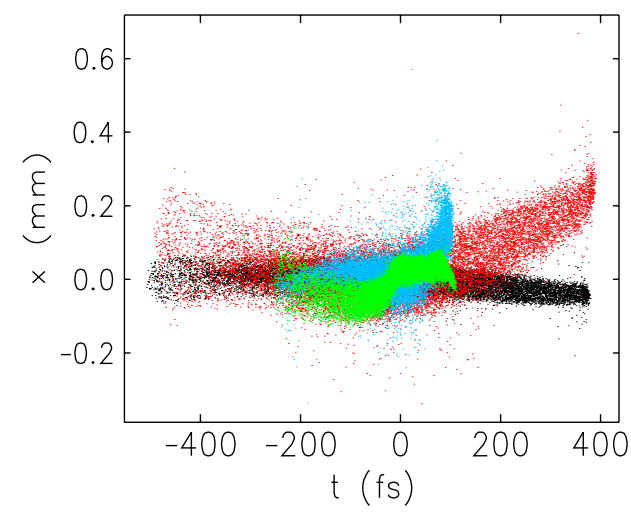

FIG. 12. Horizontal position along the bunch at $2244 \mathrm{MeV}$ (black-after linac second pass; red-after extraction; blueafter bunch compressor 3; green-after spreader and on entrance to FEL).

After the spreader the FW energy spread is reduced to $0.5 \%$ due to CSR (green).

Figure 12 shows the horizontal coordinate profile along the bunch after each transformation at $2244 \mathrm{MeV}$. It can be seen that the longitudinal compression and effects of CSR do not unduly disturb the transverse profile. This is reproduced when looking at horizontal angle and in both coordinates of the vertical plane (not shown).

\section{Final bunch properties}

Figure 13 shows the current profile at the entrance to the FEL binned in $1 \mathrm{fs}$ slices. It can be seen that $1 \mathrm{kA}$ is achieved, but varies somewhat over 100 fs. The slice emittance profile of the bunch shows that the integrity of the slices is well preserved through the machine, with all lasing slices having emittances less than $0.4 \mathrm{~mm}$ mrad. Finally, the slice energy spread profile of the bunch this is below $2 \times 10^{-4}$ for most of the lasing slices; however, at the end of the bunch this increases to $5 \times 10^{-4}$. Figure 14 shows the projected normalized emittance (less dispersive contributions) through the entire machine. It can be seen that it is well controlled, remaining below $0.4 \mathrm{~mm}$ mrad vertically and $0.6 \mathrm{~mm}$ mrad horizontally to the end of the spreader.

\section{FEL PERFORMANCE AT $1 \mathrm{KEV}$}

Simulations have been carried out to assess the performance of the NLS FEL-3 design [1] using the tracked bunch. Studies have focused on $1 \mathrm{keV}$ photon operation since this is the most demanding requirement of the FEL scheme. At this photon energy the FEL design consists of an HHG seed at $100 \mathrm{eV}$ with two stages of harmonic up-conversion to deliver enhanced bunching at the 10th harmonic of the seed at the start of a long radiator undulator tuned to $1 \mathrm{keV}$. Each stage of harmonic up-conversion consists of a modulator undulator followed by a chicane to optimize the bunching at the higher harmonic. The FEL
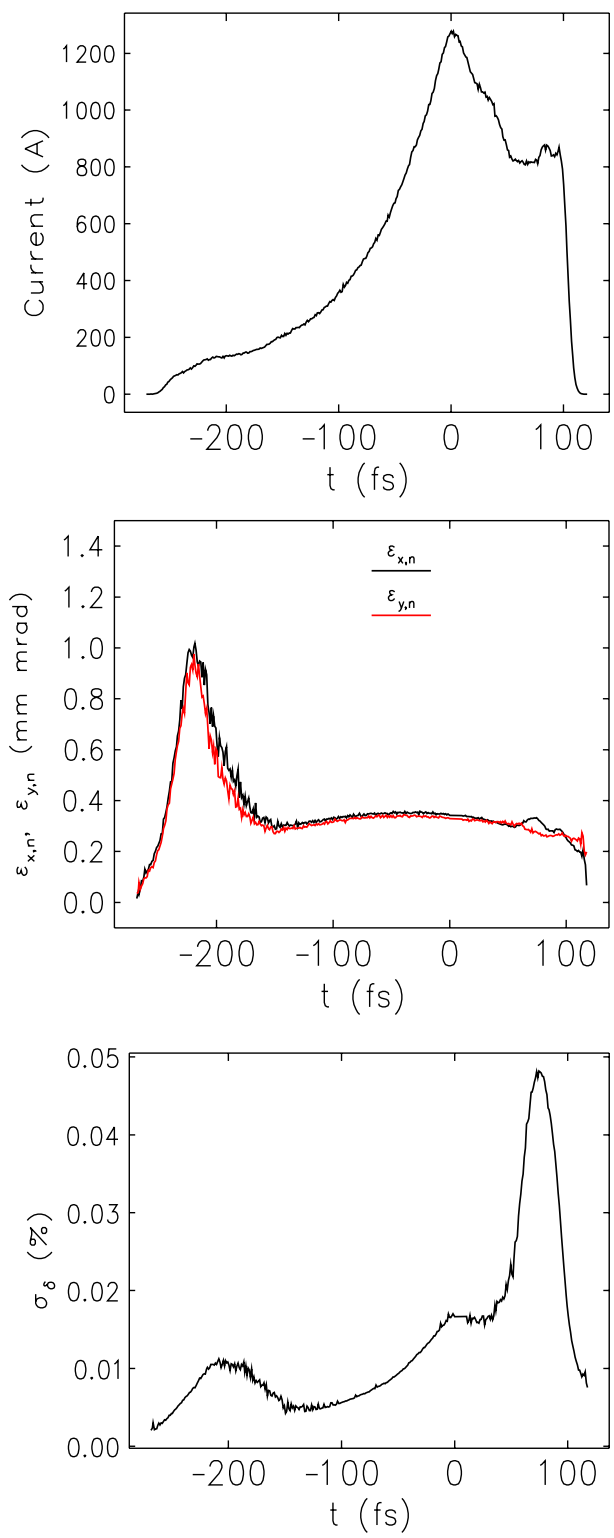

FIG. 13. Bunch properties on entrance to FEL in 1 fs slices: top-current profile; middle-slice emittance; bottom-slice energy spread.

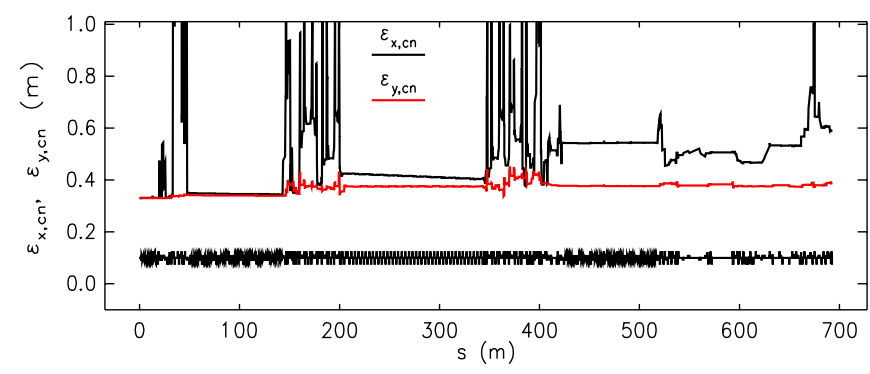

FIG. 14. Normalized emittances (less dispersive contributions) through recirculating linac to the entrance of the FEL. 


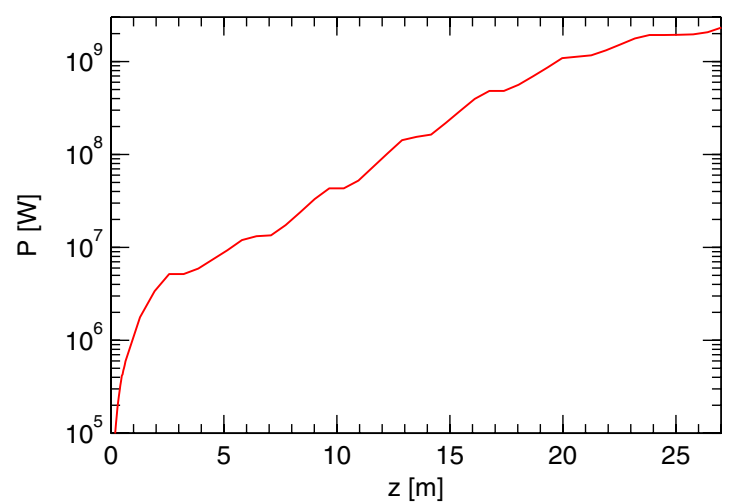

FIG. 15. Variation of peak radiation power at the fundamental with distance through the radiator for time-dependent simulations of NLS FEL-3 operating at $1 \mathrm{keV}$ photon energy.

performance was optimized for the parameters of the tracked bunch through adjusting the chicane strengths, with the steady-state mode of the standard threedimensional FEL code GENESIS 1.3 [16]. The optimal initial conditions at the start of the radiator were found to be bunching of $3 \%$ with energy spread of $0.036 \%$ rms. Timedependent FEL simulations were also carried out to provide additional information on the temporal and spectral properties. The bunching and energy spread values at the center of the seeded region were found to agree well with the steady-state results. The variation of peak radiation power at the fundamental with distance through the radiator is shown in Fig. 15. The seeded part of the bunch reaches saturation (considered to be where the radiation bandwidth is minimized) at approximately $20 \mathrm{~m}$, at which point the peak radiation output power is approximately $1 \mathrm{GW}$. The peak radiation power continues to increase beyond this point but the temporal radiation profile quality becomes degraded. The properties of the radiation output at the fundamental wavelength at saturation are shown in Fig. 16. The time-bandwidth product is close to transform limited and there are no significant discontinuities in the radiation phase in the seeded region. The contrast ratio is approximately 3600 . The FEL performance and properties of the radiation output meet the requirements of the NLS science case.

\section{CONCLUSIONS}

A recirculating linac design has been studied in detail for the NLS, motivated by the potential cost savings on both construction and operation. The proposal developed here needed many novel design concepts as well as optimization
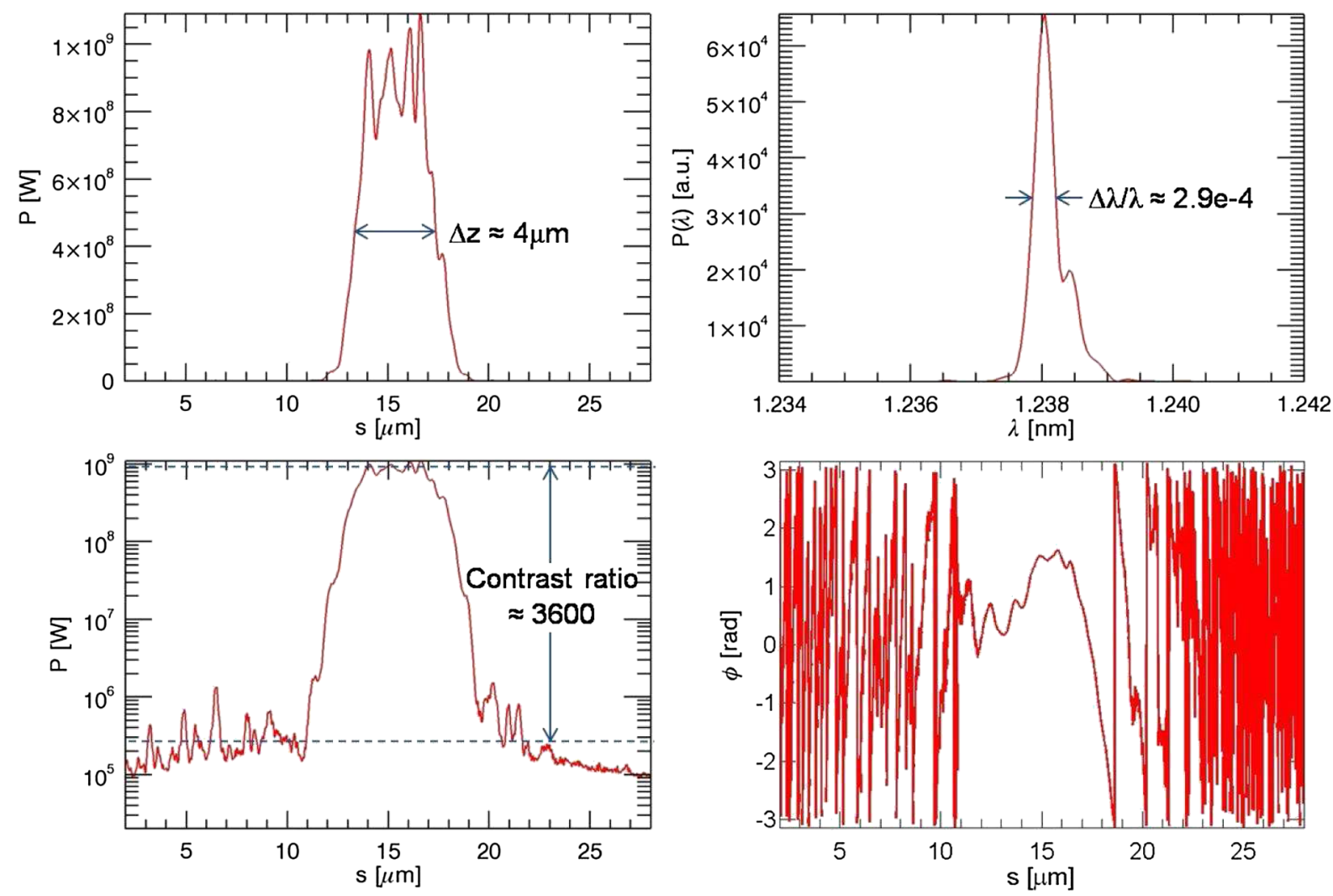

FIG. 16. Time-dependent simulation results for NLS FEL-3 at $1 \mathrm{keV}$ photon energy using the tracked bunch from the recirculating linac. Properties are plotted for a distance of $20 \mathrm{~m}$ along the radiator: Top left-longitudinal profile of FEL radiation power. Bottom left—longitudinal profile of FEL radiation power on logarithmic scale. Top right—spectrum of FEL radiation. Bottom right-radiation phase. 
procedures to be developed in order to tackle the additional features in comparison to the single pass design, and to achieve the challenging bunch parameters for seeded FELs. The design optimization is complex due to limitations arising from incoherent and coherent synchrotron radiation as well as the interplay of higher order chromatics with the energy chirp needed for bunch compression. A solution has been found which satisfies most of the design requirements for a bunch of $200 \mathrm{pC}$ charge. Start-to-end simulations have been carried out taking the output from the gun, tracking through the recirculating linac, and then calculating the FEL radiation to demonstrate that it is possible to use such a design in principle. No major show stoppers have been found for such a concept. From the present design considerations and simulations, it seems difficult to reduce the energy chirp on the final bunch; however, some ideas like using a higher-harmonic cavity after the third bunch compressor could be explored to reduce the chirp. Should this option be considered in a future detailed design stage, the optimizations should continue to reduce the energy chirp, keeping other constraints on slice emittance, slice energy spread, and peak current.

\section{ACKNOWLEDGMENTS}

We wish to thank Riccardo Bartolini, Jang-Hui Han, Ian Martin, James Rowland, and Richard Walker of Diamond Light Source Ltd., Dave Douglas of TJNAF, and Bruno Muratori and Susan Smith of Daresbury Laboratory for useful discussions.

[1] J.P. Marangos et al., New Light Source Project: Conceptual Design Report (Science \& Technology Facilities Council, 2010).
[2] The energies quoted are necessarily approximate, due to the longitudinal phase space tuning required. This involves small changes in the phases of the linac passes and in the gradient and phase of the linearizing cavities.

[3] M. Borland, Elegant: A flexible SDDS complient code for accelerator simulation, LS 287 (Advanced Photon Source, Argonne, 2000).

[4] K. L. Brown et al., SLAC-Report-91 1970.

[5] J. A. Nelder and R. Mead, Computer J. 7, 308 (1965).

[6] K. Floettmann, A space charge tracking algorithm (ASTRA), http://www.desy.de/mpyflo (DESY).

[7] J.-H. Han et al., in Proceedings of the 31st International Free Electron Laser Conference (FEL 09), Liverpool, UK (STFC Daresbury Laboratory, Warrington, 2009).

[8] Z. Huang et al., Phys. Rev. ST Accel. Beams 7, 074401 (2004).

[9] A. Meseck, M. Abo-Bakr, D. Kramer, B. Kuske, and S. Reiche, Nucl. Instrum. Methods Phys. Res., Sect. A 528, 577 (2004).

[10] P. H. Williams and H. L. Owen, 4GLS Note 14, Daresbury Laboratory, 2007 (unpublished).

[11] E. Chambers, Technical Report, Stanford High Energy Physics Laboratory, 1965 (unpublished).

[12] J. Rosenzweig and L. Serafini, Phys. Rev. E 49, 1599 (1994).

[13] D. Angal-Kalinin et al., in Proceedings of the 1st International Particle Accelerator Conference, 2010, Kyoto, Japan (IPAC'10 Organizing Committee/Asian Committee for Future Accelerators, Kyoto, 2010) [http:// accelconf.web.cern.ch/AccelConf/IPAC10/].

[14] This relative timing jitter is expected to be dominated by the absolute timing jitter of the electron bunch. In turn this will be dominated by rf amplitude and phase errors, especially in the injector. An estimate of timing jitter was conducted for the injector and was found to be 15 fs rms [7].

[15] R. Luus and T. Jaakola, AIChE J. 19, 760 (1973).

[16] S. Reiche, Nucl. Instrum. Methods Phys. Res., Sect. A 429, 243 (1999). 